\title{
Thomson scattering measurements in the divertor region of the TCV Tokamak plasmas
}

\author{
P. Blanchard*1, Y. Andrebe ${ }^{1}$, H, Arnichand ${ }^{1}$, R. Agnello ${ }^{1}$, S. Antonioni ${ }^{1}$, S. Couturier ${ }^{1}$, \\ J. Decker ${ }^{1}$, T. De Kerchove D‘Exaerde ${ }^{1}$, B.P. Duval ${ }^{1}$, I. Furno ${ }^{1}$, P.-F. Isoz ${ }^{1}$, P. \\ Lavanchy $^{1}$, X. Llobet ${ }^{1}$, B. Marlétaz ${ }^{1}$, J. Masur ${ }^{1}$ and the TCV team ${ }^{2}$ \\ ${ }^{1}$ École Polytechnique Fédérale de Lausanne (EPFL), Swiss Plasma Center (SPC), CH- \\ 1015 Lausanne, Switzerland \\ ${ }^{2}$ See author list of S. Coda et al 2019 Nucl. Fusion 59112023 \\ *Presenting author: patrick.blanchard@epfl.ch
}

\begin{abstract}
In order to better study conventional and alternative divertor configurations, the TCV Tokamak was recently upgraded. In-vessel graphite baffles were installed in the TCV chamber to delimit a separate divertor chamber from the core that required enhanced diagnostic capabilities.

To date, TCV's Thomson scattering diagnostic provided electron temperature and density profiles of the hot core and the pedestal of the plasma with an array of 96 polychromators. To study the new divertor region, an upgrade added 20 new polychromators specifically to diagnose the plasma in the relatively cold divertor region. New 4-channel polychromators were designed to provide $\mathrm{T}_{\mathrm{e}}$ measurements down to $\sim 1 \mathrm{eV}$ with $\mathrm{n}_{\mathrm{e}} \sim 1.5 \times 10^{19} \mathrm{~m}^{-3}$. Interference filters of only $1.9 \mathrm{~nm}$ FWHM were required to achieve this goal.

$T_{e}$ and $n_{e}$ measurements from the TCV divertor region have been obtained for the first time. Specific diverted plasma discharges where the outer divertor leg is scanned in and out of the TS laser beam's path are used to demonstrate the diagnostic's new capabilities and potential with measurements of $T_{e}$ as low as $1.4 \mathrm{eV}$ obtained for electron densities of $2 \times 10^{18} \mathrm{~m}^{-3}$. The system's $16 \mathrm{~mm}$ integration length along the laser line is providing, for the first time, $\mathrm{T}_{\mathrm{e}}$ and $\mathrm{n}_{\mathrm{e}}$ profiles with sufficient spatial resolution across the divertor legs to resolve the divertor plasma dynamics.
\end{abstract}

\section{Introduction}

The Swiss medium-size Tokamak à Configuration Variable (TCV), with major radius of $0.88 \mathrm{~m}$ and toroidal field $<1.45 \mathrm{~T}$, is an extremely flexible device to study tokamak physics towards fusion power plant development. Thanks to an array of specific shaping magnetic coils it can easily achieve various magnetic configurations and shapes. It is strongly contributing to the study of alternative magnetic divertor configurations such as snowflake, $\mathrm{X}$ or super-X divertor. 
In 2017 an ambitious upgrade[1] of the TCV was initiated to enhance its capability to study the divertor physic in advanced regimes of relevance for ITER and DEMO. The main part of the upgrade has been to install graphite baffles in the lower part of the TCV vacuum vessel to form a divertor chamber of variable dimensions. In complement, TCV diagnostics have been upgraded to adapt to this new configuration, especially the Thomson scattering diagnostic.

Since the beginning of its operation in 1992, TCV has been equipped with an incoherent Thomson scattering (TS) diagnostic to provide electron temperature $T_{e}$ and density $n_{e}$ measurements of the plasma core. Recent TS upgrade have successfully increased the number of observation volumes from 33 until 2013[2] to 89[3] in 2017 with increased spatial resolution down to $6 \mathrm{~mm}$ along the laser line in the pedestal. Although the plasma core was completely diagnosed, the divertor region was not. Attempts to measure $T_{e}$ and $n_{e}$ in the divertor region have been made in the past on TCV[4] but did not provide routinely TS measurements due to poor SNR and optical arrangement. The present upgrade project was therefore initiated to enhance the present TS system aiming at providing $T_{e}$ and $n_{e}$ measurement in the divertor chamber down to $\mathrm{T}_{\mathrm{e}} \sim 1 \mathrm{eV}$ for $\mathrm{n}_{\mathrm{e}} \sim 1.5 \times 10^{19} \mathrm{~m}^{-3}$ with spatial resolution of $16 \mathrm{~mm}$ on 20 new lines of sight (LOS).

A description of the TS upgrade is done in the next chapter with specific details on the interference filter development. First plasma measurements from the divertor chamber for diverted plasma will be presented in chapter 3 demonstrating this new diagnostic potential for future divertor physic studies.

\section{Divertor Upgrade}

So far the TCV TS system[3] provided measurements of $T_{e}$ and $n_{e}$ in the core region of the plasmas were temperatures are generally higher than 10-20eV. As shown in Fig. 1, 89 LOS (blue lines) are diagnosing the plasma core. Three $1.5 \mathrm{~J} \mathrm{Nd}$ :YAG lasers are fired at $20 \mathrm{~Hz}$ into the TCV vessel either independently, thus increasing the time resolution to $60 \mathrm{~Hz}$, or simultaneously for low density plasmas. Wide angle collection optic system $(\mathrm{f} / \#=2.2$, magnification=4) is imaging arrays of $1.5 \mathrm{~mm}$ diameter glass optical fibres, that are collecting the TS spectra, onto the laser path thus defining observation volumes of $6 \mathrm{~mm}$ diameter along the laser line. Specific 4- or 5-channels polychromators designed for $\mathrm{T}_{\mathrm{e}}>6 \mathrm{eV}$ are analyzing the collected TS spectra. Out of the 89 LOS, 69 consist of 2 optical fibers per polychromators and 20 (bottom blue LOS in Fig.1) have one single fibre to achieve approximately 1\% of the minor radius resolution in the pedestal region. Finally, one can recall that we are using 20ns width 
gate integrators with base-line subtraction of the scattered signals to provide one measurements points per APD and per laser pulse.

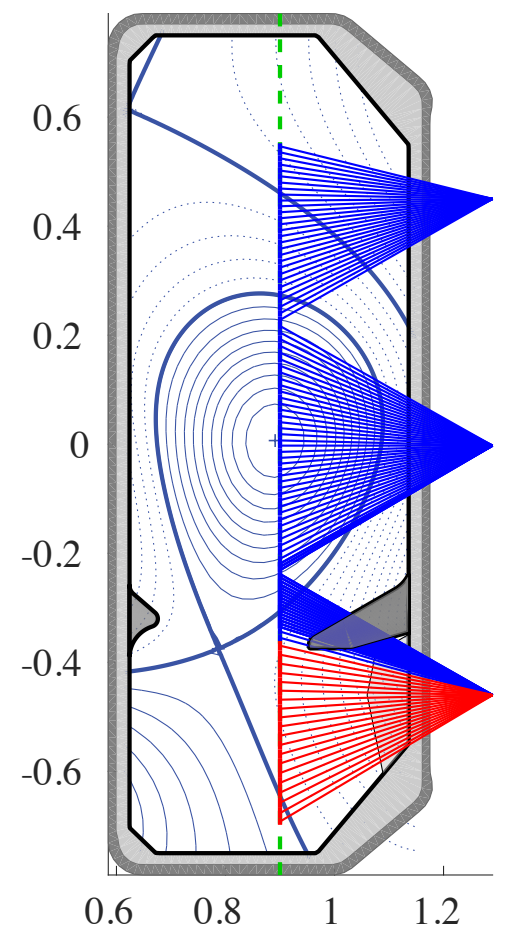

Figure 1: TCV plasma cross-section for the diverted plasma shot \#63379. The baffles defining the new divertor chamber are visible in grey. The blue LOS are the 89 core TS channels and the red LOS show the newly installed 20 channels of the divertor TS

To upgrade the TS system with divertor channels, we manufactured 4-channel compact polychromators, based on the MAST-TS[6] design, identical to the ones recently installed on TCV[3][5]. With this optimized design, the first mirror is the only moveable optical element, all the remaining optics being fixed. The collected light entering each polychromator is first directed to a high-pass filter at $830 \mathrm{~nm}$ cutoff to remove short wavelength noise. The light is then reflected by the first mirror that directs the light onto the first interference filter at an angle of incidence of $4.5^{\circ}$. At the filter, the corresponding spectral band is focused onto an Avalanche Photo-Diode (APD) while the remaining spectra is reflected back to a concave mirror that reflects the light onto the second filter and so on. Low noise and high gain APDs have been used as detectors. Sixty $1.5 \mathrm{~mm}$ diameter glass fibres have been added to our existing collection optic system. To ensure sufficient photon statistic, groups of 3 fibres each collect the light from TCV and sent it to each polychromator thus defining a spatial resolution along the laser line of about $16 \mathrm{~mm}$. The defined lines of sights are shown in red in Fig.1. 
Performing TS measurements in the divertor region of Tokamak plasma presents a number of challenges. Outside the Last Close Flux Surface (LCFS), electron temperatures are expected to drop quickly to temperature as low as few $\mathrm{eV}$ with densities much lower than the plasma core. For such experimental conditions, photon statistics such as the number of photons received by the APD detectors, the expected background noise and the TS spectrum widths are of particular importance for the design of the interference filters.

\begin{tabular}{|c|c|c|}
\hline Filter \# & CWL [nm] & FWHM [nm] \\
\hline 1 & 1061.5 & 1.9 \\
\hline 2 & 1058.4 & 6.4 \\
\hline 3 & 1048.5 & 19 \\
\hline 4 & 1025 & 40 \\
\hline
\end{tabular}

Table 1: 4-channel divertor polychromators final interference filter specifications: $\mathrm{CWL}=$ Central WaveLength: FWHM = bandwidth at Full Width Half Maximum

The final interference filter choice, as described in Table 1, is a compromise between the spatial resolution along the laser line, the lower achievable temperature of $1 \mathrm{eV}$, the number of spectral channels and the gradients of the signal ratios between channels. Fig.2(Left) shows the theoretical transmission curves of the filters.
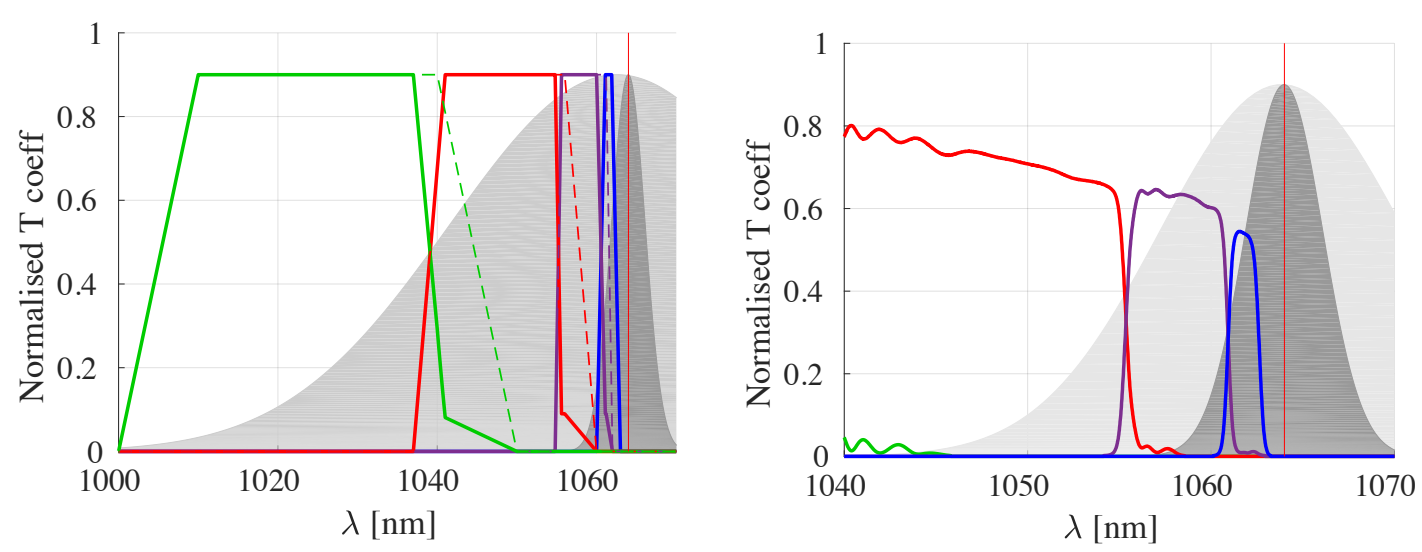

Figure 2. Normalized transmission coefficient for the TS upgrade interference filters. Left: Theoretical specifications. Dashed lines show the individual transmission for each filter, full lines show the effective filter band transmission as installed in the polychromators. The grey spectra show TS simulated spectra for $1 \mathrm{eV}$ and $100 \mathrm{eV}$. Right: calibrated spectra for the first 3 channels of polychromator \#153. The grey spectra show TS simulated spectra for $1 \mathrm{eV}$ and $10 \mathrm{eV}$.

Overlapped filter bands have been preferred to avoid losing photons between channels. The grey spectra show the expected TS spectra at $1 \mathrm{eV}$ and $100 \mathrm{eV}$. It is therefore straightforward that ultra-narrow first interference filter is required to be able to infer $T_{e}$ from $1 \mathrm{eV}$ plasmas. In 
our case, the first filter has a 1.9nm FWHM with a bandwidth of $2.25 \mathrm{~nm}$ at $10 \%$ of transmittivity. Fig.2(right) shows calibrated spectra of the first 3 channels of one of our final polychromators with the expected TS spectra at $1 \mathrm{eV}$ and $10 \mathrm{eV}$ for comparison. To avoid laser stray light at $1064 \mathrm{~nm}$, optical density greater than 5 have been achieved at the laser wavelength for all filters and anti-reflection coating has been applied. Measurements from the manufacturer show in-band transmissivity is higher than $95 \%$ for all filters.

Simulations of the of the Signal to Noise Ratio (SNR) between the number of photons received by the polychromators APD and the expected background noise levels have been made (see Fig.3 top) to optimize the interference filters selection. From our existing TS system experience and based on our simulations, we know that $\mathrm{SNR}>5$ is the minimum value to be able to infer, with sufficient precision, $\mathrm{T}_{\mathrm{e}}$ and $\mathrm{n}_{\mathrm{e}}$ from raw signals. In theory, the present filter array should be able to provide precise measurements in the $\mathrm{T}_{\mathrm{e}}$ range from $1 \mathrm{eV}$ to $500 \mathrm{eV}$. Since the signal ratio method is at the very origin of TS analysis, ratios with sufficient gradients in the $\mathrm{T}_{\mathrm{e}}$ region of interest are necessary to minimize the error in the temperature calculation. Fig. 3 middle and bottom plots show that we have sufficient ratio gradients in the $T_{e}$ range of interest and corresponding errors as low as $10 \%$ should be achievable for $\mathrm{T}_{\mathrm{e}}<5 \mathrm{eV}$.

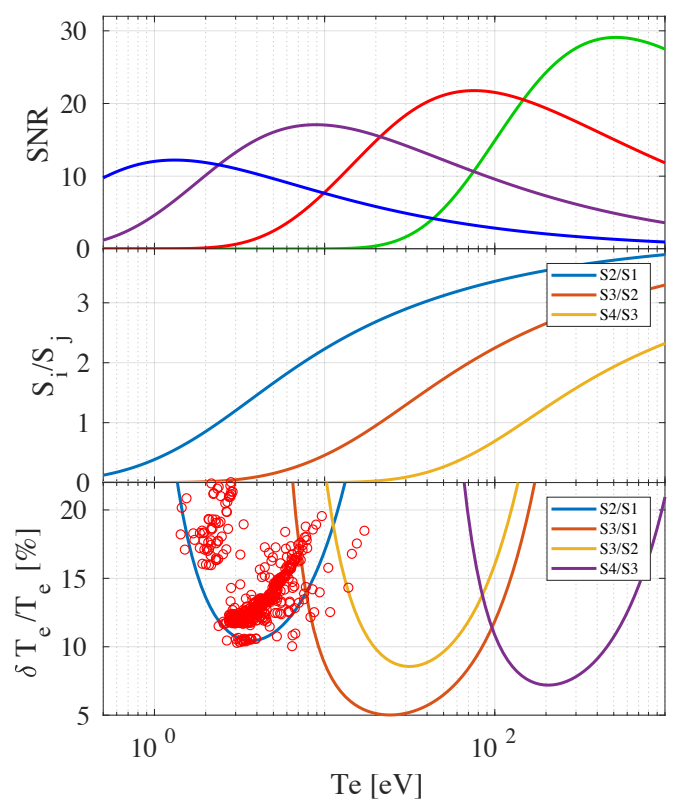

Figure 3. Top: SNR simulations vs. $\mathrm{T}_{\mathrm{e}}$ for the 4 spectral channels of the polychromator \#153 for $1.5 \mathrm{~J}$ laser, $\mathrm{n}_{\mathrm{e}}=1.5 \times 10^{19} \mathrm{~m}^{-3}$ and $16 \mathrm{~mm}$ observation volumes. Middle: Corresponding signal ratios. Bottom: Expected error in $\mathrm{T}_{\mathrm{e}}$ for selected ratios. The red dots show the TS experimental error on $\mathrm{T}_{\mathrm{e}}$ obtained for shot 63637 from the divertor channels. 


\section{Divertor Thomson scattering measurements}

Commissioning of the system was performed over the period June-August 2019. With first laser injections into TCV, stray light was observed in only one out of the 80 APD channels confirming that our laser optical path is well optimized against stray light and that the filters rejection at the laser wavelength is indeed sufficiently high. Noise levels without plasmas are of the order of $0.4 \mathrm{mV}$ after the preamplifier.
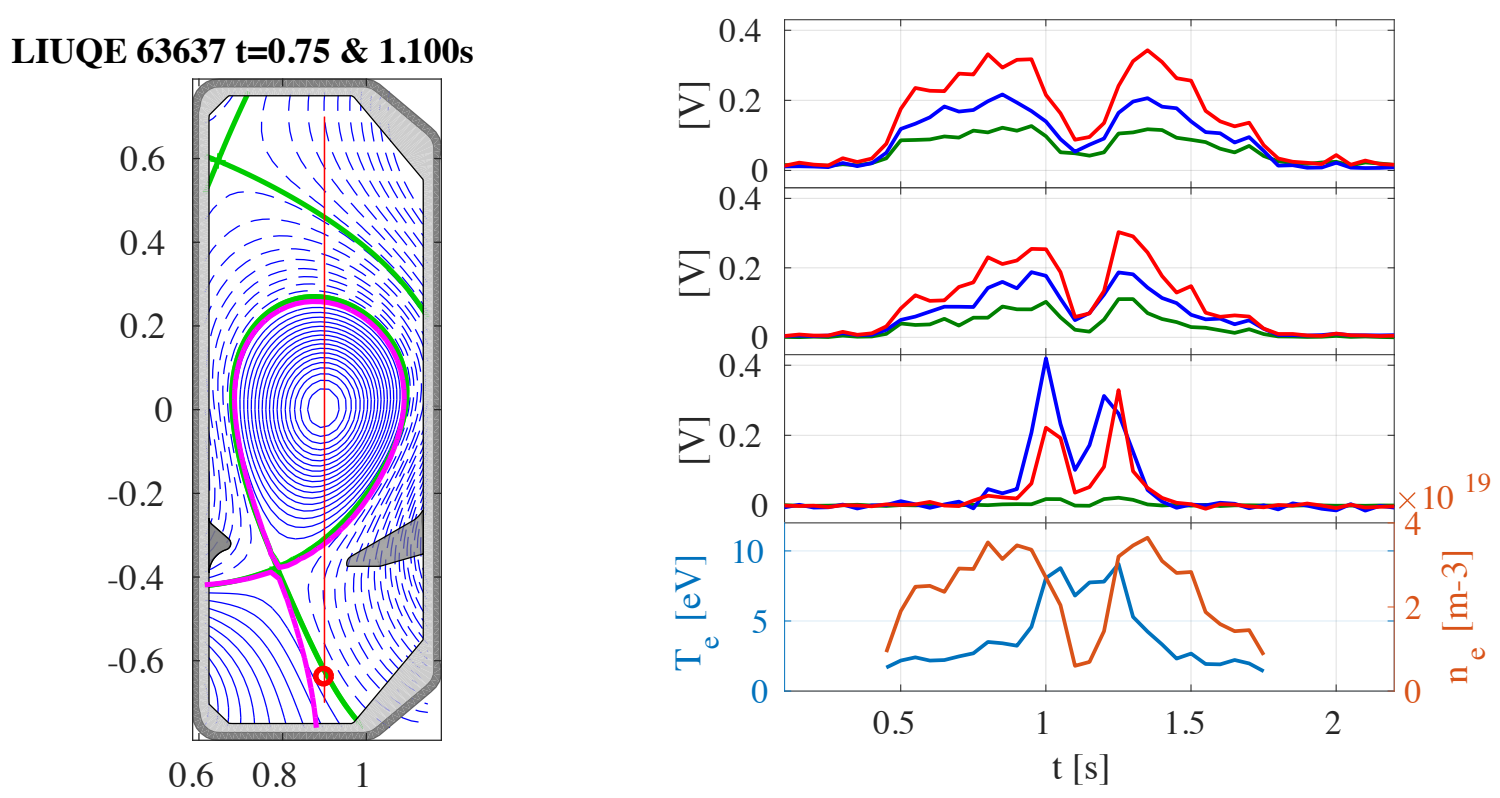

Figure 4. Left: Plasma magnetic equilibrium for diverted TCV shot $\# 63637$ at $0.75 \mathrm{~s}$ (magenta) and $1.1 \mathrm{~s}$ (green). The red circle shows the position of the polychromator \#166 which measurements are shown on the right. Right: Time evolution of TS signal amplitudes after the preamplifier in the first 3 spectral channels (top to bottom). Red, blue and green curves indicate measurements done with respectively 3,2 or $11.5 \mathrm{~J}$ laser injected simultaneously. The bottom plot shows the corresponding $\mathrm{T}_{\mathrm{e}}$ and $\mathrm{n}_{\mathrm{e}}$ temporal evolution calculated from the red curves.

Raman calibration was successfully performed and first measurements on plasmas are shown in Fig.4. In that TCV shot, a diverted plasma was created which divertor leg was swept from left to right and back from 0.5 to $1.7 \mathrm{~s}$ allowing the leg to pass over the polychromator \#166 observation volume. The shot was repeated with 1,2 and 3 simultaneous laser injection to study the effect of the laser power on the photon statistic. Temporal evolution of raw signals from the first 3 spectral channels clearly show, first, that signals are well above the noise levels and second that the laser power has indeed a crucial role in the photon statistic as expected. With only one laser, signals in the $3^{\text {rd }}$ spectral channel is at the noise level and cannot be exploited thus increasing the error in $\mathrm{T}_{\mathrm{e}}$. A strong correlation between the divertor leg position and the TS signals confirms that the overall electron density increases when the leg approaches the laser and almost completely disappear after crossing. Calculated $\mathrm{T}_{\mathrm{e}}$ and $\mathrm{n}_{\mathrm{e}}$ confirm simulation 
prediction that electron density strongly increases close to the divertor leg. Temperatures of $1.4 \pm 0.27 \mathrm{eV}$ for densities as low as $2 \pm 0.5 \times 10^{18} \mathrm{~m}^{-3}$ have been obtained for the shot with 3 lasers which confirms that the filter design allows $T_{e}$ measurements well below $2 \mathrm{eV}$ even at very low densities. Comparison between simulations and experimental $\delta \mathrm{T}_{\mathrm{e}} / \mathrm{T}_{\mathrm{e}}$ calculated with the ratio method, as shown on Fig.3 bottom, indicates very good correspondence.

In TS theory, the Salpeter parameter $\alpha$ is used to make distinction between the coherent regime $(\alpha>>1)$ and the incoherent regime $(\alpha<<1)$. Since $\alpha$ scales with $\sqrt{n_{e} / T_{e}}, \alpha$ calculations were performed from experimental results and showed $\alpha=0.05$ for $\mathrm{T}_{\mathrm{e}}=2 \mathrm{eV}$ and $\mathrm{n}_{\mathrm{e}}=2 \times 10^{19} \mathrm{~m}^{-3}$ confirming that the incoherent regime does apply to our experimental conditions.

$\mathrm{T}_{\mathrm{e}}$ and $\mathrm{n}_{\mathrm{e}}$ profiles for a diverted plasma are shown in Fig.5 and compared to the observation volume positions. The full potential of those new polychromators diagnosing the divertor chamber is well illustrated by the clear $n_{\mathrm{e}}$ spatial resolution outside of the separatrix. Electron densities of the same order of magnitude as in the plasma core are measured close to the divertor legs while electron temperatures are decreasing below $10 \mathrm{eV}$ in the divertor region. TCV has a powerful diagnostic with sufficient spatial resolution and accessible $T_{e}$ range to resolve the divertor plasma dynamics.
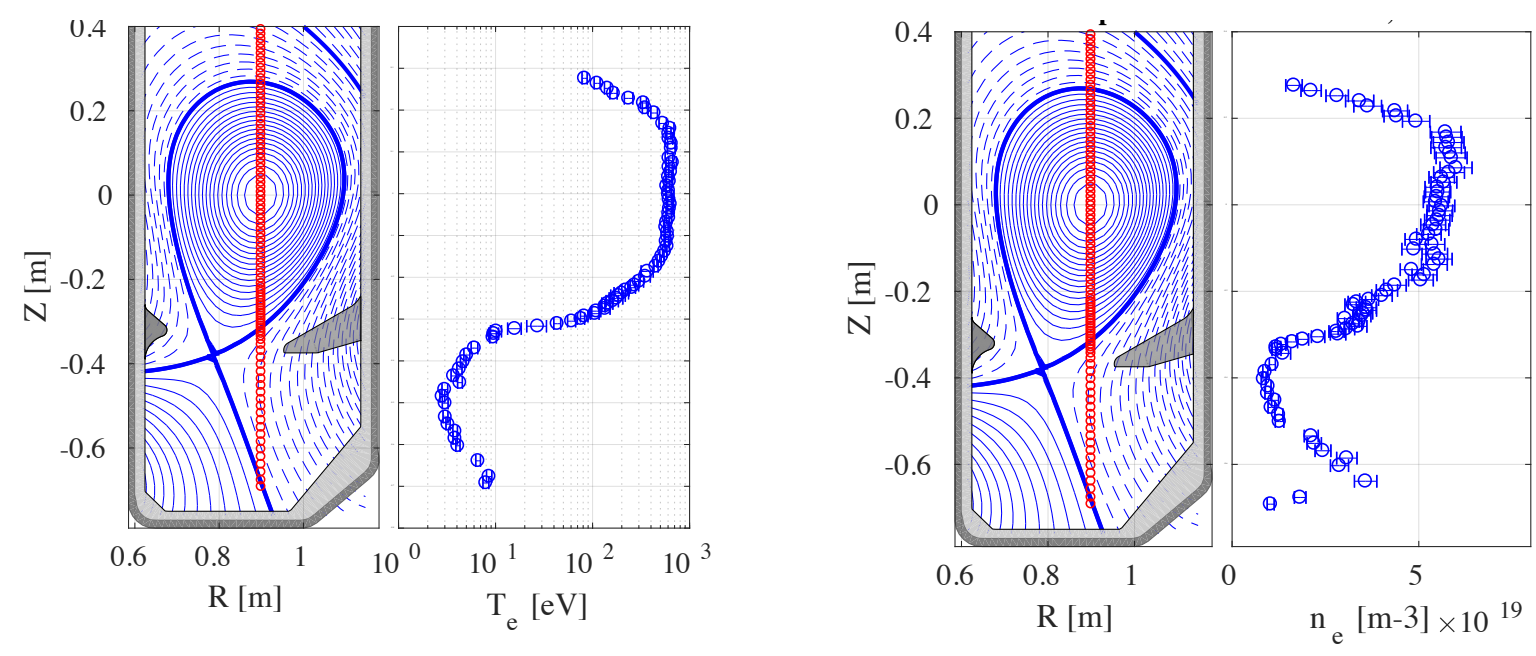

Figure 5. $\mathrm{T}_{\mathrm{e}}$ (left) and $\mathrm{n}_{\mathrm{e}}$ (right) profiles for shot 63638 at $\mathrm{t}=0.95 \mathrm{~s}$ compared to the $\mathrm{TS}$ observation volumes central positions (red circles) and plasma magnetic equilibrium

\section{Conclusions}

The TCV TS diagnostic has been successfully upgraded with an additional 20 lines of sight diagnosing the divertor chamber of TCV. 4-channels polychromators fitted with ultra-narrow 
interference filters of $1.9 \mathrm{~nm}$ FWHM have demonstrated their capability to measure $\mathrm{T}_{\mathrm{e}}$ below $1.5 \mathrm{eV}$ for densities as low as $2 \times 10^{18} \mathrm{~m}^{-3}$ without being affected by laser stray light. This upgrade provides TCV with a diagnostic capable to give a new insight into the divertor physics main parameters with a spatial resolution of $16 \mathrm{~mm}$.

\section{Acknowledgments}

This work has been carried out within the framework of the EUROfusion Consortium and has received funding from the Euratom research and training program 2014-2018 and 2019-2020 under grant agreement No 633053. The views and opinions expressed herein do not necessarily reflect those of the European Commission. This work was supported in part by the Swiss National Science Foundation.

\section{References}

[1] H. Reimerdes et al 2017 Nuclear Materials and Energy 121106

[2] A. Pitzschke, Pedestal Characteristics and MHD Stability of H-mode Plasmas in TCV, EPFL Thesis \#4917 (2011)

[3] J. Hawke et al 2017 JINST 12 C12005

[4] R. Behn et al 1999 RSI 70768 (1999)

[5] H. Arnichand et al 2019 JINST 14 C09013

[6] R. Scannell et al., Rev. Sci. Instr. 79, 10E730, (2008) 